\title{
AHL-priming functions via oxylipin and salicylic acid
}

\author{
Sebastian T. Schenk and Adam Schikora* \\ Institute for Phytopathology, Research Centre for Biosystems, Land Use and Nutrition (IFZ), Justus Liebig University Giessen, Giessen, Germany
}

\section{Edited by:}

Hua Lu, University of Maryland Baltimore County, USA

Reviewed by:

Anton Hartmann, Helmholtz Zentrum München-German Research Center for Environmental Health, Germany Shuishan Song, Biology Institute Hebei Academy of Sciences, China

\section{*Correspondence:}

Adam Schikora, Institute for Phytopathology, Research Centre for Biosystems, Land Use and Nutrition (IFZ), Justus Liebig University Giessen, Heinrich-Buff-Ring 26-32, 35392 Giessen, Germany e-mail: adam.schikora@agrar. uni-giessen.de
Collaborative action between the host plant and associated bacteria is crucial for the establishment of an efficient interaction. In bacteria, the synchronized behavior of a population is often achieved by a density-dependent communication called quorum sensing. This behavior is based on signaling molecules, which influence bacterial gene expression. $\mathrm{N}$-acyl homoserine lactones (AHLs) are such molecules in many Gram-negative bacteria. Moreover, some AHLs are responsible for the beneficial effect of bacteria on plants, for example the long chain N-3-oxo-tetradecanoyl-L-homoserine lactone (oxo-C14$\mathrm{HSL}$ ) can prime Arabidopsis and barley plants for an enhanced defense. This AHL-induced resistance phenomenon, named AHL-priming, was observed in several independent laboratories during the last two decades. Very recently, the mechanism of priming with oxoC14-HSL was shown to depend on an oxylipin and salicylic acid (SA). SA is a key element in plant defense, it accumulates during different plant resistance responses and is the base of systemic acquired resistance. In addition, SA itself can prime plants for an enhanced resistance against pathogen attack. On the other side, oxylipins, including jasmonic acid (JA) and related metabolites, are lipid-derived signaling compounds. Especially the oxidized fatty acid derivative cis-OPDA, which is the precursor of JA, is a newly described player in plant defense. Unlike the antagonistic effect of SA and JA in plant-microbe interactions, the recently described pathway functions through a synergistic effect of oxylipins and $\mathrm{SA}$, and is independent of the JA signaling cascade. Interestingly, the oxo-C14-HSLinduced oxylipin/SA signaling pathway induces stomata defense responses and cell wall strengthening thus prevents pathogen invasion. In this review, we summarize the findings on AHL-priming and the related signaling cascade. In addition, we discuss the potential of AHL-induced resistance in new strategies of plant protection.

Keywords: AHL, quorum sensing (QS), oxylipins, SA, priming

\section{AHLS IMPACT ON PLANT PHYSIOLOGY AND DEVELOPMENT}

Cell-to-cell signaling is a widespread practice in living organisms. Bacteria use a pheromone-like system called quorum sensing (QS). QS was first described in Vibrio fischeri, a bacterium that lives in symbiosis with a squid and produces bioluminescent light at high cell densities (Tomasz, 1965; Kempner and Hanson, 1968; Ruby and Nealson, 1976). V. fischeri produces $N$-acyl homoserine lactones (AHLs) that are freely diffusible across the bacterial membranes and accumulate in their surroundings (Kempner and Hanson, 1968). When a threshold concentration of AHLs is achieved, the bacterial population is able to sense (sensing) the critical cell density, the so-called quorum (Tomasz, 1965). Besides a regulation of the AHL-regulon, a very important feature of QS is the autoinduction of AHL-synthase expression. This communication system enables individual bacterial cells to monitor the population density and coordinate a conjoint action(s) (Waters and Bassler, 2005; Antunes and Ferreira, 2009; Teplitski et al., 2010; Nazzaro etal., 2013). In many situations, the ability of bacterial population to behave co-operatively and to communicate with each other brings clear advantages; for example, bacteria benefit from QS for conjugation, symbiotic, or pathogenic interactions with the host, for adaptation and distribution within an ecological niche (efficiency sensing; Hense et al., 2007), or for the production and secretion of secondary metabolites like antibiotics or siderophores (Williams, 2007; Hartmann etal., 2014). In Gram-negative bacteria, QS system is often based on AHLs, it is until now the best characterized bacterial communication system (Engebrecht and Silverman, 1984). AHL molecules can vary in the length of the acyl chain (4-18-carbons) and in the substitutions at the carbon chain. In addition to AHLs, 2-alkyl-4-quinolones, long-chain fatty acids, fatty acid methyl esters, and furanones (autoinducer-2) can be used for bacterial communication (Williams, 2007).

Reports from independent laboratories claimed that the short chain AHLs induce a growth promotion effect due to an impact on the phytohormone auxin (von Rad etal., 2008; Bai etal., 2012; Liu et al., 2012). The first study of AHL impact on plant hormone metabolism was performed with Medicago truncatula during the response to AHLs originated from the symbiotic bacterium Ensifer meliloti (Sinorhizobium meliloti). In this study, authors revealed 150 differentially regulated proteins, within those were several auxin-induced proteins and enzymes that are involved in auxin metabolism. Furthermore, the activation of the $\beta$-Glucuronidase (GUS) reporter gene under the control of the auxin-responsive $G H 3$ promoter, indicated the involvement of auxin in the response to AHL (Mathesius et al., 2003). The 
possible role of auxin in response to AHL treatment was also suggested by transcriptional analyses. Auxin-associated genes were induced after a treatment with the short chain $N$-hexanoylhomoserine lactone (C6-HSL) as well as after a pretreatment with the long chain $\mathrm{N}$-3-oxo-tetradecanoyl-L-homoserine lactone (oxo-C14-HSL) and a subsequent challenge with the pathogen elicitor flg22 (von Rad et al., 2008; Schenk et al., 2014). In addition, genes involved in cytokinin metabolism, which have an antagonistic function to auxin, were down regulated (von Rad et al., 2008). The same report described an alteration of the free auxin to cytokinin ratio in root and shoot tissues after AHL application, explaining as a consequence the promotion of plant growth (von Rad et al., 2008). Another study showed the involvement of auxin in the AHL-induced growth as a result of the production of hydrogen peroxide and nitric oxide, which are dependent on the cyclic GMP signaling. In the postulated model, the QS molecule $\mathrm{N}$-3-oxo-dodecanoyl-L-homoserine lactone (oxo-C10HSL), induced an enhanced basipetal auxin transport followed by accumulation of $\mathrm{H}_{2} \mathrm{O}_{2}$ and $\mathrm{NO}$, and stimulated therefore the formation of adventitious roots (Bai et al., 2012). Nevertheless, some publications disagree with the involvement of auxin in the AHLgrowth promoting effect on plants. Despite the strong impact of oxo-C10-HSL on primary root growth and in contrast to other findings, lateral root formation, and root hair development was independent of auxin signaling as indicated by the expression analysis of the GUS-reporter genes under the control of the auxinregulated DR5 promoter (Ortiz-Castro et al., 2008). Moreover, a recent report suggested that the growth promoting effect of AHLs depends on the AHL-derivative L-homoserine, which is produced upon amidolysis of AHLs by the fatty acid amide hydrolase (Palmer et al., 2014). The authors postulated that the increased transpiration induced by L-homoserine, would enhance the water and minerals flow through plant organism and therefore positively influence the growth.

Beside the enhancement of growth, long chain AHLs have impact on plant defense mechanisms (Schikora et al., 2011; Schenk et al., 2012, 2014). In contrast to animals, plants do not have specialized cells for immune responses; for this reason, the attacked plant cell needs to reprogram its regular cellular functions for a defense response. Plants developed specialized local defense mechanisms and specific systemic responses, which are coordinated by systemic signals (Spoel and Dong, 2008). In this coordination, the cross talk between hormones plays a crucial role (Koornneef and Pieterse, 2008). The defense response against necrotrophic pathogens is usually dependent on the plant hormones jasmonic acid (JA) and ethylene (ET), while the defense reactions to biotrophic pathogens are dominantly regulated by salicylic acid (SA; Glazebrook, 2005). The antagonistic interaction between the SA and JA is well characterized (Rojo et al., 2003; Beckers and Spoel, 2006), although some reports claim a synergistic interaction between these two phytohormones (van Wees et al., 2000). An involvement of defense hormones in the AHL-induced resistance was postulated after the observation that an inoculation with the AHL-producing rhizobacterium Serratia liquefaciens strain MG1 enhanced systemic defense and the accumulation of SA in tomato plants (Hartmann et al., 2004; Schuhegger et al., 2006). Similar results were observed after a treatment of tomato plants with pure C6- and C4-HSL; the SAand ET-dependent Pathogenesis Related1a (PR1a) and two chitinase genes were highly expressed after the treatment (Schuhegger et al., 2006). The enhanced expression of those genes in tomato leaves after application of C6-HSL or C4-HSL to the roots suggested that the systemic response functions via an SA-dependent pathway (Schuhegger et al., 2006). Likewise, an application of the long chain AHL (oxo-C14-HSL) on Arabidopsis roots induced a systemic response in plant shoots (Schikora et al., 2011). The AHLinduced pathway could therefore depend on SA together with the oxylipin 12-oxo-phytodienoic acid (cis-OPDA), as indicated by the accumulation of those two hormones, as well as mutant studies and transcriptional analyses (Schenk et al., 2014), see also chapter on AHL-priming below.

\section{THE SYNERGISTIC ROLE OF SA AND OXYLIPINS IN PLANT DEFENSE}

Phyto-oxylipins are a diverse group of lipid-derived compounds including JA and jasmonate-related metabolites like cis-OPDA, methyl jasmonate, and the active form of JA, jasmonyl- $l$-isoleucine (JA-Ile). These compounds are unsaturated fatty acids produced by lipoxygenases (LOX) that oxidize the lipid chain at the C9 or C13 position (Andreou and Feussner, 2009). Additionally, oxylipins can be synthesized non-enzymatically via the free radical-catalyzed pathway, which generates similar structures denominated phytoprostanes (Sattler et al., 2006). While a lot is known about the biological function of JA, methyl jasmonate, and JA-Ile, including their perception and signal transduction (reviewed in Browse, 2009), the biological role of oxylipins before their conversion to JA is less understood. Nevertheless, several studies assumed that the precursors of JA play a role in different developing processes and during defense responses (Blee, 2002; Dave and Graham, 2012). For example, 18-cabon divinyl ether fatty acid, colneleic, and colnelenic acids accumulated in potato and tobacco leaves during the late blight disease (Weber et al., 1999). In addition, phytoprostanes accumulated as a consequence of pathogen-induced oxidative stress (ROS-production), induced the activation of Mitogen-Activated Protein Kinases (MAPKs) and glutathione-S-transferase (GST), expression of defense genes, and the accumulation of phytoalexin (Thoma et al., 2003). Furthermore, the enzymatically oxidized cis-OPDA induced expression of genes related to detoxification, stress responses, and secondary metabolism (Taki et al., 2005; Mueller et al., 2008). Interestingly, the oxylipins-related pathways induced reactions distinct from the JA-induced responses. While the expression of JA-related genes is COI1-dependent, cis-OPDA and phytoprostanes ( $\mathrm{PPA}_{1}$ and $\left.\mathrm{PPB}_{1}\right)$ have been demonstrated to activate gene expression in a COI1independent manner (Stintzi et al., 2001; Taki et al., 2005; Stotz et al., 2013). Transcriptional analysis of Arabidopsis showed that more than 150 genes responded to the application of cis-OPDA but not to JA or methyl jasmonate (Taki et al., 2005). The expression of the majority of these genes was regulated through the bZIP TGACG motif-binding transcription factors TGA2, TGA5, and TGA6 (Stotz et al., 2013). Curiously, those transcription factors are also required for the activation of SA-dependent genes (Zhang et al., 1999, 2003). A recent discovery indicated that the oxylipin pathway induced by biotic stress interacts with the SA-dependent 
signaling and results in a stomatal defense response (Montillet et al., 2013). The authors postulated that during stomatal defense the activation of MPK3 and MPK6 induced the guard cell lipoxygenase LOX1 and hence the peroxidation of poly unsaturated fatty acids into oxylipins followed by the accumulation of SA. Downstream of this SA accumulation was the regulation of the anion channel SLAC-1, which coordinates the stomatal defense response.

\section{THE PRIMING EFFECT, SENSITIZING FOR FUTURE DEFENSE RESPONSES}

One of the consequences of an activated defense mechanism is a high consumption of energy. Therefore, the immune system of higher organisms needs to be coordinated in an efficient manner. In order to lower the cost of defense, plants developed different mechanisms to orchestrate their immune system, among them are negative regulators that suppress the defense response in the absence of a pathogen, or the induction of specific pathways, accordingly to the particular pathogen. Furthermore, plants may use priming as an efficient regulation of defense responses. This mechanism is based on a sensitization of the plant for a stronger and faster response. This phenomenon has been used in agriculture for plant protection since the early 1930s. Priming was usually defined as a part of induced resistance; however, the priming effect is only assessable after a subsequently challenge of the primed tissue (Conrath et al., 2002). Some priming inducers are well characterized, one of them is the non-proteinogenic amino acid $\beta$-aminobutyric acid (BABA) and another is SA at low concentrations. BABA priming functions through a SA- and abscisic acid (ABA)-dependent pathway, and induces enhanced callose depositions and tolerance to salt stress (Ton et al., 2005). In addition, BABA-induced resistance interferes with the action of the bacterial toxin coronatine (COR) from the pathogen Pseudomonas syringae (Tsai et al., 2011). Yet another priming inducer is the mobile metabolite azelaic acid, which induces a systemic protection via accumulation of SA (Jung et al., 2009).

Besides the accumulation of signaling components, few reports addressed the molecular mechanism of priming and explained the sensitized status of a plant. The first revealed the accumulation of the inactive form of MPK3 that can be rapidly activated upon a subsequent attack (Beckers et al., 2009). The second was the discovery of chromatin modifications on promoters of defense-related genes. In primed plants, histones in promoter regions of the defense-associated transcription factors WRKY6, WRKY26, and WRKY53 are methylated (H3Kme3 and $\mathrm{H} 3 \mathrm{~K} 4 \mathrm{me} 2)$ and acetylated (H3K9, H4K5, and $\mathrm{H} 4 \mathrm{~K} 12)$, which could explain the faster activation and the subsequent stronger stress response (Jaskiewicz et al., 2011). Interestingly, and very important for future research projects, is the fact that the primed status of a plant can be transmitted to next generations. The SA-induced defense and the resistance to the pathogen $P$. syringae were inherited to the offspring by transferring the histone methylation mechanism of relevant genes (Luna et al., 2012). Furthermore, the transgenerational priming was observed in progeny of plants treated with BABA or exposed to insect attack (Rasmann et al., 2012; Slaughter et al., 2012).
However, while BABA and P. syringe priming are based on SA and SA-depending signaling, the insect-induced transgenerational priming is JA-dependent.

\section{AHL-PRIMING DEPENDS ON AN OXYLIPIN/SA-DEPENDENT PATHWAY}

Considering that SA and JA precursors are crucial for long chain AHL-priming, the cross talk between SA and oxylipins seems to be an important feature of the AHL-induced resistance. Evidences that the AHL-induced priming acts via oxylipins/SA-dependent pathway are not restricted to the accumulation of phytohormones after sensitizing the plant with AHLs also genetic evidences support this dependency. Since the Arabidopsis mutants coil-16 and jarl behaved like wild-type plants when tested for AHL-enhanced resistance against $P$. syringae, the effect seems to be independent of the JA perception and the production of JA-Ile (Schenk et al., 2014). However, AHL-priming required Nonexpressor of PR Genes 1 (NPR1), which is the key regulator in SA-dependent defense, as indicated by the high proliferation of $P$. syringae in AHL-pretreated npr1-1 mutant plants. The same holds true for the triple mutant $\operatorname{tga} 2 / 5 / 6$, which is impaired in the signal transduction in cis-OPDA- and SA-signaling cascade(s). Likewise, the AHL effect was lost in the lox2 mutant (Schenk etal., 2014), missing the Lipoxygenase 2, one of the enzymes required for the oxidation of the unsaturated fatty acids, and hence for the oxylipin response in plants (Blee, 2002; Dave and Graham, 2012). In addition to the genetic studies, evidences that oxo-C14-HSL acts via the oxylipin/SA-induced pathway were observed at transcriptional level. For example, the enhanced expression of GST6, GSTU19, the stress responding heat shock proteins encoding genes HSP70 and HSP17, and the cytochrome P450 (CYP81D11), which was observed after a cis-OPDA treatment (Mueller et al., 2008), was also visible during AHL-priming (Schenk et al., 2014). Furthermore, the independency of JA and ET during AHL-priming was strengthened by the expression patterns of prominent JAresponsive genes, MYC2 and VSP2, and the ET-responsive genes PR3, ERF5, and ETR1, which were not influenced by the AHL pretreatment (Schenk et al., 2014).

\section{STOMATA DEFENSE RESPONSE, ONE OF THE MECHANISMS USED DURING AHL-INDUCED RESISTANCE}

Stomata are openings in the epidermal layer of terrestrial plants. These pores are built up of two guard cells that regulate the opening and closure in order to establish the exchange of gasses between the leaf and the environment. This regulation system allows the control of transpiration. During drought stress, the regulation of anion-channels in guard cells is coordinated by ABA. The perception of ABA activates the guard cell-specific, ABA-related protein kinase OST1, which is followed by production of ROS and activation of $\mathrm{Ca}^{2+}$-signaling (Mustilli et al., 2002). Moreover, stomatal closure is tightly controlled by innate immunity as it has a crucial role during prevention of pathogen invasion. This phenomenon is referred to as stomatal defense response and functions as physical barrier against pathogen entry (Melotto et al., 2008).

A report on the inoculation of Arabidopsis plants with $P$. syringae showed that guard cells perceive the pathogen, indicating 
an active role of guard cells in plant defense (Melotto et al., 2006). However, the guard cells response to biotic stress seems to differ from the response to abiotic stress in respect to the function of the plant defense hormones SA and ABA, as well as the MAP kinases and NPR1 (Melotto et al., 2006; Zeng and $\mathrm{He}, 2010)$. Even though, ABA and SA signaling pathways were apparently involved in the stomatal closure induced by the beneficial bacterium Bacillus subtilis FB17 (Kumar et al., 2012), the above discussed report on ABA-independent pathway that controls stomatal closure in case of an immune defense response, proposed a signaling pathway, which is induced upon the perception of flg22 and includes the activation of MPK3 and MPK6 (Montillet etal., 2013). The authors observed that it requires the guard-cell-specific LOX1, producing the oxylipin cis-OPDA. A high accumulation of cis-OPDA after the flg22-elicitation in guard cells was followed by an accumulation of SA (Montillet et al., 2013).

Interestingly, the AHL-induced resistance also depends on SA and cis-OPDA and activates the stomatal defense response (Figure 1). We observed an increase of closed and reduction of open stomata in oxo-C14-HSL-pretreated Arabidopsis plants
(Schenk et al., 2014). Furthermore, the expression profile of ABAdependent genes $R D 22, R D 29$, and $R A B 18$ revealed no regulation in oxo-C14-HSL-primed plants, which strengthens the postulated hypothesis on ABA-independency in stomatal defense response (Montillet et al., 2013; Schenk et al., 2014).

An important feature of phytopathogenic bacteria is the ability to reopen closed stomata, thus counteract the stomatal defense response. This is usually achieved by the bacterial toxin coronatine (COR) (Melotto et al., 2006; Zeng and He, 2010; Pieterse etal., 2014), which mimics the plant hormone JA-Ile. This virulence factor binds to the JA-receptor complex and activates the antagonistic crosstalk between SA and JA, inhibiting the flg22-triggered immune responses such as ROS production and callose depositions (Yi etal., 2014). In addition, COR suppresses the biosynthesis and accumulation of SA, hence inhibits the local and systemic defense responses (Zheng et al., 2012). Interestingly, the priming agent BABA interferes with the COR impact on stomatal defense responses. BABAinduced resistance activates the SA-dependent responses, while COR was able to suppress this defense reactions as shown by the abolished BABA effect by relatively high concentration of

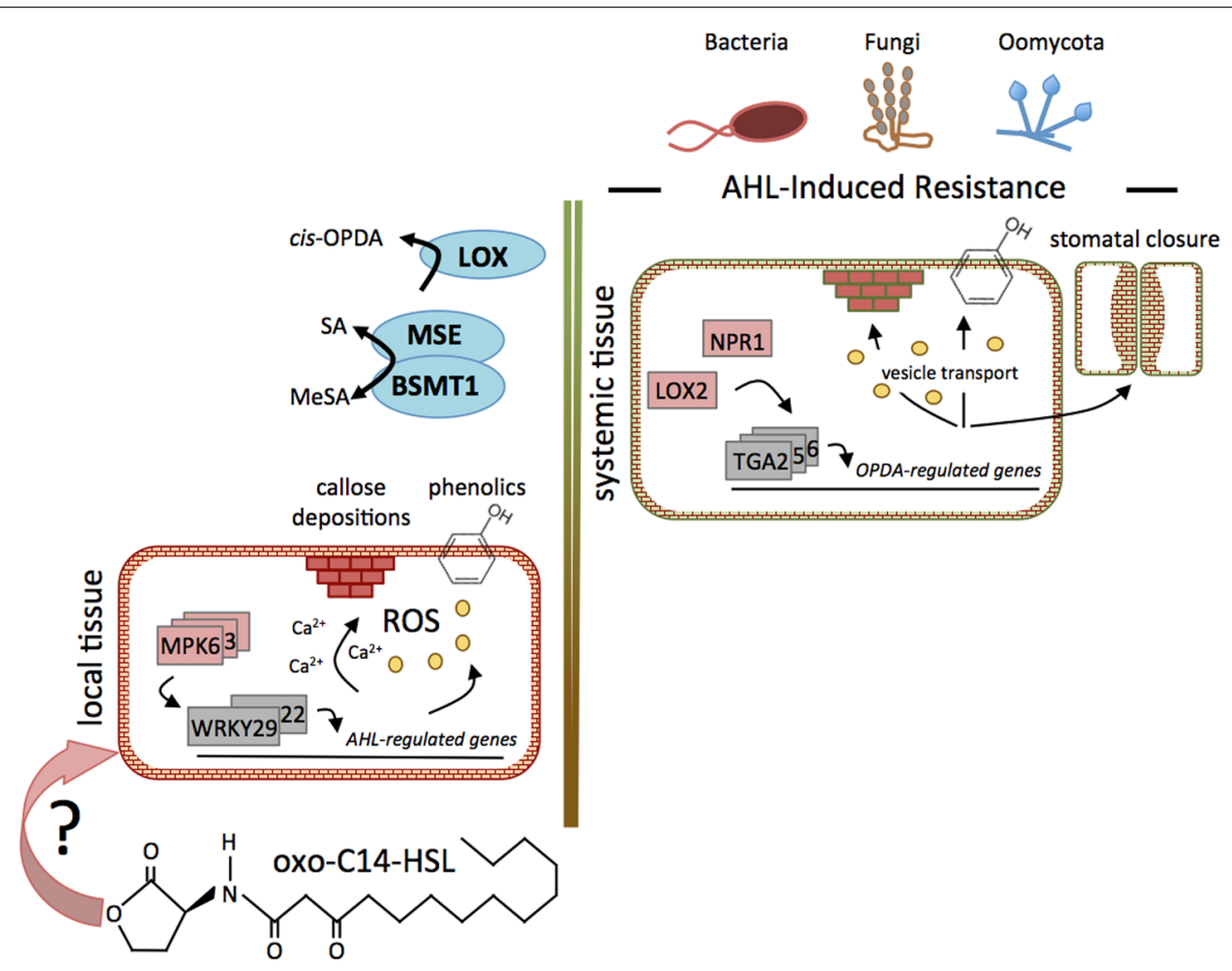

FIGURE 1 | Function of SA and oxylipins in AHL-induced priming. Signaling steps of AHL-induced mechanisms leading to the reinforcement of resistance against several pathogens. The perception mechanism(s) of AHL in plant tissues is not known, indicated by "?". Nonetheless, in local tissues of Arabidopsis plants, the oxo-C14-HSL-priming is manifested through the prolonged and stronger activation of MAPKs and the enhanced expression of WRKY transcription factors, followed by transcriptional reprogramming of genes related to $\mathrm{Ca}^{2+}$-signaling, defense, G-proteins, cell wall, and flavonoid metabolism. Furthermore, AHLs induced a higher accumulation of ROS, phenolic compounds, and callose in the cell walls. Even though long chain AHLs are not translocated to distal tissues, elevated production of the phytohormones oxylipin (cis-OPDA) and SA was observed in distal tissues, indicating that a systemic signaling is involved in this phenomenon. The proteins NPR1 and LOX2, as well as the TGA2/5/6 transcription factors were required. Like in the local tissue, the long chain AHL oxo-C14-HSL induced callose depositions, accumulation of phenolic compounds, and enhanced stomatal closure. 
COR, or the failure to prime the coil-16 mutant (Tsai et al., 2011). Similarly, in the case of AHL-induced resistance the stomatal defense response seems to depend on SA and could disrupt the function of COR. However, the AHL-priming is still present in the coil-16 mutant, which indicates differences between the BABA- and AHL-priming (Schenk etal., 2014).

\section{AGRICULTURAL POTENTIAL OF AHL-PRIMING}

Since the beginning of the twenty-first century, the bivalence between avoidance of synthetic pesticides and the performance of crop protection methods is a big challenge in agriculture. To ensure a sufficient food supply, agriculture industry has to develop modern plant protection strategies, which ensure sufficient yield and food quality. Moreover, due to market demands, farmers are under increasing pressure to produce their crops organically, or at least to reduce the chemical impact on the environment. In addition, plant production has to deal with ecological challenges like abiotic or biotic stresses and handle the arable land in the most sustainable manner. Development of new substances, which are useful in both integrated agricultural management and organic farming, is a big challenge. The development of biologicals or biocontrol agents, which originate from natural products, could be a possible strategy to meet those requirements. For instance the use of microbial inoculants of beneficial, soil-born microorganisms could be a competent approach to support agriculture (Berg, 2009). Using the knowledge of microbe-plant interactions, rhizosphere, or root-associated bacteria including Bacillus, Pseudomonas, and Serratia spp. could contribute to the production of new natural products for plant protection (Berg, 2009; Beneduzi et al., 2012; Nadeem et al., 2013). Likewise, microbial metabolites with an impact on plant growth or health have a high potential in this regard (Brader et al., 2014). The bacterial QS molecules are remarkable candidates in such strategies (Hartmann et al., 2014). Purified QS molecules and bacteria with increased production of AHLs, have an impact on plant defense mechanisms and portrait the agricultural potential of homoserine lactones (Zarkani et al., 2013; Hernández-Reyes et al., 2014). Furthermore, the use of $\mathrm{N}_{2}$-fixating Rhizobia, with their positive effects on plant physiology, could be improved by QS molecules. Nodulation efficiency, symbiosome development, exopolysaccharides production, nitrogen fixation, and adaptation to stress are all regulated by QS systems (Gonzalez and Marketon, 2003; Marketon et al., 2003). The promotion of AHL production in Rhizobia or bacterial inoculants could enhance the beneficial effects (nitrogen fixation, growth promotion, reinforced plant defense) hence, lead to a reduced use of fertilizers or conventional plant protection agents in agriculture, and in this way lower the negative impact of chemicals on the environment. Another strategy was proposed by two independent laboratories, which have bioengineered tobacco and tomato plants with different bacterial AHL-synthesis genes. These transgenic plants foster beneficial plant-bacteria interactions, and alter growth and tolerance to abiotic stress (Scott et al., 2006; Barriuso et al., 2008). However, risks and advantages of AHL-producing plants need to be assessed and require further elucidation.

\section{REFERENCES}

Andreou, A., and Feussner, I. (2009). Lipoxygenases - Structure and reaction mechanism. Phytochemistry 70, 1504-1510. doi: 10.1016/j.phytochem.2009. 05.008

Antunes, L. C., and Ferreira, R. B. (2009). Intercellular communication in bacteria. Crit. Rev. Microbiol. 35, 69-80. doi: 10.1080/10408410902733946

Bai, X., Todd, C. D., Desikan, R., Yang, Y., and Hu, X. (2012). N-3-Oxo-DecanoylL-Homoserine-Lactone activates auxin-induced adventitious root formation via hydrogen peroxide- and nitric oxide-dependent cyclic GMP signaling in mung bean. Plant Physiol. 158, 725-736. doi: 10.1104/pp.111.185769

Barriuso, J., Ramos Solano, B., Fray, R. G., Camara, M., Hartmann, A., and Gutierrez Manero, F. J. (2008). Transgenic tomato plants alter quorum sensing in plant growth-promoting rhizobacteria. Plant Biotechnol. J. 6, 442-452. doi: 10.1111/j.1467-7652.2008.00331.x

Beckers, G. J., Jaskiewicz, M., Liu, Y., Underwood, W. R., He, S. Y., Zhang, S., et al. (2009). Mitogen-activated protein kinases 3 and 6 are required for full priming of stress responses in Arabidopsis thaliana. Plant Cell 21, 944-953. doi: 10.1105/tpc.108.062158

Beckers, G. J., and Spoel, S. H. (2006). Fine-tuning plant defence signalling: salicylate versus jasmonate. Plant Biol. (Stuttg.) 8, 1-10. doi: 10.1055/s-2005872705

Beneduzi, A., Ambrosini, A., and Passaglia, L. M. (2012). Plant growth-promoting rhizobacteria (PGPR): their potential as antagonists and biocontrol agents. Genet. Mol. Biol. 35, 1044-1051. doi: 10.1590/S1415-47572012000600020

Berg, G. (2009). Plant-microbe interactions promoting plant growth and health: perspectives for controlled use of microorganisms in agriculture. Appl. Microbiol. Biotechnol. 84, 11-18. doi: 10.1007/s00253-009-2092-7

Blee, E. (2002). Impact of phyto-oxylipins in plant defense. Trends Plant Sci. 7, 315-322. doi: 10.1016/S1360-1385(02)02290-2

Brader, G., Compant, S., Mitter, B., Trognitz, F., and Sessitsch, A. (2014). Metabolic potential of endophytic bacteria. Curr. Opin. Biotechnol. 27, 30-37. doi: 10.1016/j.copbio.2013.09.012

Browse, J. (2009). Jasmonate passes muster: a receptor and targets for the defense hormone. Annu. Rev. Plant Biol. 60, 183-205. doi: 10.1146/annurev.arplant.043008.092007

Conrath, U., Pieterse, C. M., and Mauch-Mani, B. (2002). Priming in plantpathogen interactions. Trends Plant Sci. 7, 210-216. doi: 10.1016/S13601385(02)02244-6

Dave, A., and Graham, I. A. (2012). Oxylipin signaling: a distinct role for the jasmonic acid precursor cis-(+)-12-Oxo-Phytodienoic Acid (cis-OPDA). Front. Plant Sci. 3:42. doi: 10.3389/fpls.2012.00042

Engebrecht, J., and Silverman, M. (1984). Identification of genes and gene products necessary for bacterial bioluminescence. Proc. Natl. Acad. Sci. U.S.A. 81, 41544158. doi: 10.1073/pnas.81.13.4154

Glazebrook, J. (2005). Contrasting mechanisms of defense against biotrophic and necrotrophic pathogens. Annu. Rev. Phytopathol. 43, 205-227. doi: 10.1146/annurev.phyto.43.040204.135923

Gonzalez, J. E., and Marketon, M. M. (2003). Quorum sensing in nitrogen-fixing rhizobia. Microbiol. Mol. Biol. Rev. 67, 574-592. doi: 10.1128/MMBR.67.4.574592.2003

Hartmann, A., Gantner, S., Schuhegger, R., Steidle, A., Dürr, C., Schmid, M., et al. (2004). "N-acyl homoserine lactones of rhizosphere bacteria trigger systemic resistance in tomato plants," in Proceedings of the 11th International Congress on Molecular Plant-Microbe Interactions, eds B. Lugtenberg, I. Tikhonovich, and N. Provorov (St. Paul, MN: APS Press), 554-556.

Hartmann, A., Rothballer, M., Hense, B. A., and Schröder, P. (2014). Bacterial quorum sensing compounds are important modulators of microbe-plant interactions. Front. Plant Sci. 5:131. doi: 10.3389/fpls.2014.00131

Hense, B. A., Kuttler, C., Mueller, J., Rothballer, M., Hartmann, A., and Kreft, J. U. (2007). Opinion - Does efficiency sensing unify diffusion and quorum sensing? Nat. Rev. Microbiol. 5, 230-239. doi: 10.1038/nrmicro1600

Hernández-Reyes, C., Schenk, S. T., Neumann, C., Kogel, K. H., and Schikora, A. (2014). N-acyl-homoserine lactones-producing bacteria protect plants against plant and human pathogens. Microb. Biotechnol. 7, 580-588. doi: 10.1111/17517915.12177

Jaskiewicz, M., Conrath, U., and Peterhansel, C. (2011). Chromatin modification acts as a memory for systemic acquired resistance in the plant stress response. EMBO Rep. 12, 50-55. doi: 10.1038/embor.2010.186 
Jung, H. W., Tschaplinski, T. J., Wang, L., Glazebrook, J., and Greenberg, J. T. (2009). Priming in systemic plant immunity. Science 324, 89-91. doi: $10.1126 /$ science. 1170025

Kempner, E. S., and Hanson, F. E. (1968). Aspects of light production by Photobacterium fischeri. J. Bacteriol. 95, 975-979.

Koornneef, A., and Pieterse, C. M. (2008). Cross talk in defense signaling. Plant Physiol. 146, 839-844. doi: 10.1104/pp.107.112029

Kumar, A. S., Lakshmanan, V., Caplan, J. L., Powell, D., Czymmek, K. J., Levia, D. F., et al. (2012). Rhizobacteria Bacillus subtilis restricts foliar pathogen entry through stomata. Plant J. 72, 694-706. doi: 10.1111/j.1365-313X.2012.05116.x

Liu, F., Bian, Z., Jia, Z., Zhao, Q., and Song, S. (2012). The GCR1 and GPA1 participate in promotion of Arabidopsis primary root elongation induced by $\mathrm{N}$-acyl-homoserine lactones, the bacterial quorum-sensing signals. Mol. Plant Microbe Interact. 25, 677-683. doi: 10.1094/MPMI-1011-0274

Luna, E., Bruce, T. J., Roberts, M. R., Flors, V., and Ton, J. (2012). Nextgeneration systemic acquired resistance. Plant Physiol. 158, 844-853. doi $10.1104 /$ pp.111.187468

Marketon, M. M., Glenn, S. A., Eberhard, A., and Gonzalez, J. E. (2003). Quorum sensing controls exopolysaccharide production in Sinorhizobium meliloti. J. Bacteriol. 185, 325-331. doi: 10.1128/JB.185.1.325-331.2003

Mathesius, U., Mulders, S., Gao, M., Teplitski, M., Caetano-Anolles, G., Rolfe, B. G., et al. (2003). Extensive and specific responses of a eukaryote to bacterial quorum-sensing signals. Proc. Natl. Acad. Sci. U.S.A. 100, 1444-1449. doi: 10.1073/pnas.262672599

Melotto, M., Underwood, W., and He, S. Y. (2008). Role of stomata in plant innate immunity and foliar bacterial diseases. Annu. Rev. Phytopathol. 46, 101-122. doi 10.1146/annurev.phyto.121107.104959

Melotto, M., Underwood, W., Koczan, J., Nomura, K., and He, S. Y. (2006). Plant stomata function in innate immunity against bacterial invasion. Cell 126, 969 980. doi: 10.1016/j.cell.2006.06.054

Montillet, J. L., Leonhardt, N., Mondy, S., Tranchimand, S., Rumeau, D., Boudsocq, M., et al. (2013). An abscisic acid-independent oxylipin pathway controls stomatal closure and immune defense in Arabidopsis. PLoS Biol. 11:e1001513. doi: 10.1371/journal.pbio. 1001513

Mueller, S., Hilbert, B., Dueckershoff, K., Roitsch, T., Krischke, M., Mueller, M. J., et al. (2008). General detoxification and stress responses are mediated by oxidized lipids through TGA transcription factors in Arabidopsis. Plant Cell 20, 768-785. doi: 10.1105/tpc.107.054809

Mustilli, A. C., Merlot, S., Vavasseur, A., Fenzi, F., and Giraudat, J. (2002). Arabidopsis OST1 protein kinase mediates the regulation of stomatal aperture by abscisic acid and acts upstream of reactive oxygen species production. Plant Cell 14, 3089-3099. doi: 10.1105/tpc.007906

Nadeem, S. M., Ahmad, M., Zahir, Z. A., Javaid, A., and Ashraf, M. (2013). The role of mycorrhizae and plant growth promoting rhizobacteria (PGPR) in improving crop productivity under stressful environments. Biotechnol. Adv. 32, 429-448. doi: 10.1016/j.biotechadv.2013.12.005

Nazzaro, F., Fratianni, F., and Coppola, R. (2013). Quorum sensing and phytochemicals. Int. J. Mol. Sci. 14, 12607-12619. doi: 10.3390/ijms140612607

Ortiz-Castro, R., Martinez-Trujillo, M., and Lopez-Bucio, J. (2008). Nacyl-L-homoserine lactones: a class of bacterial quorum-sensing signals alter post-embryonic root development in Arabidopsis thaliana. Plant Cell Environ. 31, 1497-1509. doi: 10.1111/j.1365-3040.2008. 01863.x

Palmer, A. G., Senechal, A. C., Mukherjee, A., Ane, J. M., and Blackwell, H. E. (2014). Plant responses to bacterial N-acyl L-homoserine lactones are dependent on enzymatic degradation to L-homoserine. ACS Chem. Biol. 9, 1834-1845. doi: 10.1021/cb500191a

Pieterse, C. M. J., Zamioudis, C., Berendsen, R. L., Weller, D. M., Van Wees, S. C. M., and Bakker, P. A. H. M. (2014). Induced systemic resistance by beneficial microbes. Annu. Rev. Phytopathol. 52, 347-375. doi: 10.1146/annurev-phyto082712-102340

Rasmann, S., De Vos, M., Casteel, C. L., Tian, D., Halitschke, R., Sun, J. Y., etal. (2012). Herbivory in the previous generation primes plants for enhanced insect resistance. Plant Physiol. 158, 854-863. doi: 10.1104/pp.111. 187831

Rojo, E., Solano, R., and Sanchez-Serrano, J. J. (2003). Interactions between signaling compounds involved in plant defense. J. Plant Growth Regul. 22, 82-98. doi: $10.1007 /$ s00344-003-0027-6
Ruby, E. G., and Nealson, K. H. (1976). Symbiotic association of Photobacterium fischeri with the marine luminous fish Monocentris japonica; a model of symbiosis based on bacterial studies. Biol. Bull. 151, 574-586. doi: 10.2307/15 40507

Sattler, S. E., Mene-Saffrane, L., Farmer, E. E., Krischke, M., Mueller, M. J., and Dellapenna, D. (2006). Nonenzymatic lipid peroxidation reprograms gene expression and activates defense markers in Arabidopsis tocopherol-deficient mutants. Plant Cell 18, 3706-3720. doi: 10.1105/tpc.106.044065

Schenk, S. T., Hernandez-Reyes, C., Samans, B., Stein, E., Neumann, C., Schikora, M., et al. (2014). N-acyl-homoserine lactone primes plants for cell wall reinforcement and induces resistance to bacterial pathogens via the salicylic acid/oxylipin pathway. Plant Cell 26, 2708-2723. doi: 10.1105/tpc.114. 126763

Schenk, S. T., Stein, E., Kogel, K. H., and Schikora, A. (2012). Arabidopsis growth and defense are modulated by bacterial quorum sensing molecules. Plant Signal. Behav. 7, 178-181. doi: 10.4161/psb.18789

Schikora, A., Schenk, S. T., Stein, E., Molitor, A., Zuccaro, A., and Kogel, K. H. (2011). N-acyl-homoserine lactone confers resistance towards biotrophic and hemibiotrophic pathogens via altered activation of AtMPK6. Plant Physiol. 157, 1407-1418. doi: 10.1104/pp.111.180604

Schuhegger, R., Ihring, A., Gantner, S., Bahnweg, G., Knappe, C., Vogg, G., et al. (2006). Induction of systemic resistance in tomato by $\mathrm{N}$-acyl-L-homoserine lactone-producing rhizosphere bacteria. Plant Cell Environ. 29, 909-918. doi: 10.1111/j.1365-3040.2005.01471.x

Scott, R. A., Weil, J., Le, P. T., Williams, P., Fray, R. G., Von Bodman, S. B., et al. (2006). Long- and short-chain plant-produced bacterial N-acyl-homoserine lactones become components of phyllosphere, rhizosphere, and soil. Mol. Plant Microbe Interact. 19, 227-239. doi: 10.1094/MPMI-19-0227

Slaughter, A., Daniel, X., Flors, V., Luna, E., Hohn, B., and Mauch-Mani, B. (2012). Descendants of primed Arabidopsis plants exhibit resistance to biotic stress. Plant Physiol. 158, 835-843. doi: 10.1104/pp.111.191593

Spoel, S. H., and Dong, X. (2008). Making sense of hormone crosstalk during plant immune responses. Cell Host Microbe 3, 348-351. doi: 10.1016/j.chom. 2008.05.009

Stintzi, A., Weber, H., Reymond, P., Browse, J., and Farmer, E. E. (2001). Plant defense in the absence of jasmonic acid: the role of cyclopentenones. Proc. Natl. Acad. Sci. U.S.A. 98, 12837-12842. doi: 10.1073/pnas.211311098

Stotz, H. U., Mueller, S., Zoeller, M., Mueller, M. J., and Berger, S. (2013). TGA transcription factors and jasmonate-independent COI1 signalling regulate specific plant responses to reactive oxylipins. J. Exp. Bot. 64, 963-975. doi: 10.1093/jxb/ers389

Taki, N., Sasaki-Sekimoto, Y., Obayashi, T., Kikuta, A., Kobayashi, K., Ainai, T., et al. (2005). 12-oxo-phytodienoic acid triggers expression of a distinct set of genes and plays a role in wound-induced gene expression in Arabidopsis. Plant Physiol. 139, 1268-1283. doi: 10.1104/pp.105.067058

Teplitski, M., Mathesius, U., and Rumbaugh, K. P. (2010). Perception and degradation of $\mathrm{N}$-acyl homoserine lactone quorum sensing signals by mammalian and plant cells. Chem. Rev. 111, 100-116. doi: 10.1021/cr100045m

Thoma, I., Loeffler, C., Sinha, A. K., Gupta, M., Krischke, M., Steffan, B., et al. (2003). Cyclopentenone isoprostanes induced by reactive oxygen species trigger defense gene activation and phytoalexin accumulation in plants. Plant J. 34, 363-375. doi: 10.1046/j.1365-313X.2003.01730.x

Tomasz, A. (1965). Control of the competent state in pneumococcus by a hormonelike cell product: an example for a new type of regulatory mechanism in bacteria. Nature 208, 155-159. doi: 10.1038/208155a0

Ton, J., Jakab, G., Toquin, V., Flors, V., Iavicoli, A., Maeder, M. N., et al. (2005). Dissecting the beta-aminobutyric acid-induced priming phenomenon in Arabidopsis. Plant Cell 17, 987-999. doi: 10.1105/tpc.104.029728

Tsai, C. H., Singh, P., Chen, C. W., Thomas, J., Weber, J., Mauch-Mani, B., et al. (2011). Priming for enhanced defence responses by specific inhibition of the Arabidopsis response to coronatine. Plant J. 65, 469-479. doi: 10.1111/j.1365313X.2010.04436.x

van Wees, S. C., De Swart, E. A., van Pelt, J. A., van Loon, L. C., and Pieterse, C. M. (2000). Enhancement of induced disease resistance by simultaneous activation of salicylate- and jasmonate-dependent defense pathways in Arabidopsis thaliana. Proc. Natl. Acad. Sci. U.S.A. 97, 8711-8716. doi: 10.1073/pnas. 130425197

von Rad, U., Klein, I., Dobrev, P. I., Kottova, J., Zazimalova, E., Fekete, A., et al. (2008). Response of Arabidopsis thaliana to N-hexanoyl-DL-homoserine-lactone, 
a bacterial quorum sensing molecule produced in the rhizosphere. Planta 229, 73-85. doi: 10.1007/s00425-008-0811-4

Waters, C. M., and Bassler, B. L. (2005). Quorum sensing: cell-to-cell communication in bacteria. Annu. Rev. Cell Dev. Biol. 21, 319-346. doi: 10.1146/annurev.cellbio.21.012704.131001

Weber, H., Chételat, A., Caldelari, D., and Farmer, E. E. (1999). Divinyl ether fatty acid synthesis in late blight-diseased potato leaves. Plant Cell 11, 485-493. doi: 10.1105/tpc.11.3.485

Williams, P. (2007). Quorum sensing, communication and cross-kingdom signalling in the bacterial world. Microbiology 153, 3923-3938. doi: 10.1099/mic.0.2007/012856-0

Yi, S. Y., Shirasu, K., Moon, J. S., Lee, S. G., and Kwon, S. Y. (2014). The activated SA and JA signaling pathways have an influence on flg22-triggered oxidative burst and callose deposition. PLOS ONE 9:e88951. doi: 10.1371/journal.pone. 0088951

Zarkani, A. A., Stein, E., Rohrich, C. R., Schikora, M., Evguenieva-Hackenberg, E., Degenkolb, T., et al. (2013). Homoserine lactones influence the reaction of plants to rhizobia. Int. J. Mol. Sci. 14, 17122-17146. doi: 10.3390/ijms140 817122

Zeng, W., and He, S. Y. (2010). A prominent role of the flagellin receptor FLAGELLIN-SENSING2 in mediating stomatal response to Pseudomonas syringae pv tomato DC3000 in Arabidopsis. Plant Physiol. 153, 1188-1198. doi: 10.1104/pp.110.157016

Zhang, Y., Fan, W., Kinkema, M., Li, X., and Dong, X. (1999). Interaction of NPR1 with basic leucine zipper protein transcription factors that bind sequences required for salicylic acid induction of the PR-1 gene. Proc. Natl. Acad. Sci. U.S.A. 96, 6523-6528. doi: 10.1073/pnas.96.11.6523
Zhang, Y. L., Tessaro, M. J., Lassner, M., and Li, X. (2003). Knockout analysis of Arabidopsis transcription factors TGA2, TGA5, and TGA6 reveals their redundant and essential roles in systemic acquired resistance. Plant Cell 15, 2647-2653. doi: 10.1105/tpc.014894

Zheng, X. Y., Spivey, N. W., Zeng, W., Liu, P. P., Fu, Z. Q., Klessig, D. F., et al. (2012). Coronatine promotes Pseudomonas syringae virulence in plants by activating a signaling cascade that inhibits salicylic acid accumulation. Cell Host Microbe 11, 587-596. doi: 10.1016/j.chom.2012.04.014

Conflict of Interest Statement: The authors declare that the research was conducted in the absence of any commercial or financial relationships that could be construed as a potential conflict of interest. The reviewer, Anton Hartmann declares that, despite having collaborated with the author Adam Schikora, the review process was handled objectively and no conflict of interest exists.

Received: 13 October 2014; accepted: 17 December 2014; published online: 14 January 2015.

Citation: Schenk ST and Schikora A (2015) AHL-priming functions via oxylipin and salicylic acid. Front. Plant Sci. 5:784. doi: 10.3389/fpls.2014.00784

This article was submitted to Plant-Microbe Interaction, a section of the journal Frontiers in Plant Science.

Copyright (c) 2015 Schenk and Schikora. This is an open-access article distributed under the terms of the Creative Commons Attribution License (CC BY). The use, distribution or reproduction in other forums is permitted, provided the original author(s) or licensor are credited and that the original publication in this journal is cited, in accordance with accepted academic practice. No use, distribution or reproduction is permitted which does not comply with these terms. 